Historic, Archive Document

Do not assume content reflects current scientific knowledge, policies, or practices. 



\section{Variety and Price List SPRING 1922}

\section{Smith Bros.' Nurseries CENTERVILLE, UTAH}

I We have the following list of Fruit and Ornamental Trees, Shrubs, Vines \& Etc, to offer our patrons and friends, the same will be in effect until sold.

d All our stock will be up to the usual high standard and the same care and attention which have characterized our business for 35 years will be given every order.

d Nursery Stock of every kind is extremely limited throughout the world this season and will be harder to get as the season advances. Wisdom suggests that you book your wants early.

I Where cash accompanies order Freight or Express will be prepaid to any point in Utah, otherwise orders will read F. O. B. Centerville, Utah.

I All shipments will be made C. O. D. to parties unknown to us or who fail to furnish satisfactory references.

d We will go the limit in helping every patron make a success.

By Their Fruits Ye Shall Know Them 


\section{Listed in about their order of ripening.}

\section{PEACH}

No. 1 Grade $50 \mathrm{c}$ each $\$ 40.00$ - 100, $\$ 350.00$ - 1000

No. 2 Grade

$\$ 35.00-100, \$ 300.00$ - 1000

$\checkmark$ May Flower

- Alexander

- Red Bird Cling

Hales Early

? Carmine

Crawfords Early

Grix

Early Elberta

J. H. Hale

Elberta

Tuscan, Orange Cling Heath, White Cling

Nectrine $75 \mathrm{c}$ each

\section{APRICOT}

1 Grade $60 \mathrm{c}$ each, $\$ 50.00$ per 100

2 Grade 50c “ 40.00

Moorpark

Chinese

\section{PEAR}

75 c each, $\$ 60.00$ per 100

$\checkmark$ Bartlett

Flemish Beauty

Keifer

Bure de Anjou

NUT

S. S. Almond, 75c each Eng. W alnut, $\$ 1.50$ "

\section{APPLE}

No. 1 Grade

60 e each $\$ 50.00$ per 100

-Early Harvest

- Red June

Red Astrichan

Yellow Transparent

McIntosh Red

Wealthy

Jonathan

Banana

Winter Pearmain

Wine Sap

Delicious

Roman Beauty

Grimes Golden

Siberian Crab

\section{PLUM}

60 e each $\$ 50.00$ per 100

$\checkmark$ Peach

$\checkmark$ Burbank

Bradshaw

Wickson

Satsuma

Damson

\section{PRUNE}

60 c each $\$ 50.60$ per 100

$\checkmark$ French

Golden

Italian 


\section{SMALL FRUITS}

Fays Prolific Currant, 2yr. 25c each $\$ 20.00$ per 100

Fays Prolific

Profection,

Boskop, (Black Eng.)

Downing Gooseberry

Poorman

Chatqua

Loganberry

" 1 "6 20 "6

$35 \quad 66$

35 "6

35 " 6

35

(New) 50

20c ea $\$ 15.00$ - $100 \$ 125.00$ - 1000

Himalay a Blackberry 15

Evergreen

Snyder

Ward

Wilson Jr.

$\begin{array}{lll}15 & \text { ، } & 10 . \\ 15 & \text { ، } & 10 . \\ 15 & \text { ، } & 10 . \\ 15 & \text { “ } & 10 .\end{array}$

15.00 . "6

20.00 " "

20.00 " "

20.00 " "6

20.00 " "

\section{RASPBERRY}

Cuthbert, Red

Loudon

Malboro

Antwerp

Columbian BlackCap15

Plum Farmer, ',

Dewberry, Lucretia
$66 \quad 100 . \quad 66$

6. $75 . \quad 66$

675.66

$675 . \quad 66$

$675 . \quad 66$

\section{GRAPES}

Concord, 1 year

Concord, 2 "

10 "6 5.

10 “ 5.

10 ، 5.

$\begin{array}{lll}15 & \text { ، } 7.50\end{array}$

"6 40.

" 40.

" 40.

" 60.

60.

60.

50.

6.

15.

125.

66

Agawam

35 '6

35 ،

Niagara

35 ،

Black Pearl

35

Muscat,Cal Varieties20

" 20

Malaga

Flame Tokay " 20

Thompsons Seedless 20

Moores All Season Pie Plant 40c each

12.50 " 100.

66

12.50 " 100.

12.50

" 100.

100.

$\$ 4.00$ a doz.

\section{hter Grade Stock in Quantity.}




\section{Shade and Ornamental Trees and Shrubs}

Carolina Poplar, 10 to $12 \mathrm{ft}$. $75 \mathrm{c}$ each, $\$ 50.00$ per 100

\begin{tabular}{|c|c|c|c|c|c|}
\hline 66 & 8 ' & $10^{6}$ & 50 & 66 & 35.00 \\
\hline 66 & $6^{6}$ & 8 '، & 35 & 66 & 20.00 \\
\hline 66 & & 6 6 & 25 & 66 & 15.00 \\
\hline
\end{tabular}

Volga Poplar, 12 “" 14 “ $\$ 1.00$ "

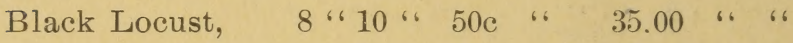

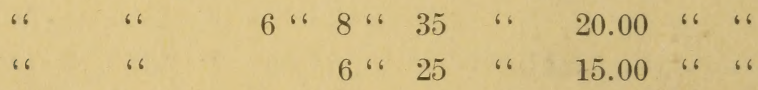

Norway Maple 8 " 810 "\$2.00 "

Black Walnut 7 " 8 “ 1.00 “

\section{Flowering Shrubs}

Spira, Van Houttei, (Bridlewreath) 2 to $3 \mathrm{ft}$. $75 \mathrm{c}$ each Spira, Thunbergi

Barberry

Snowballs

Lilacs, (White and Purple)

Forsythia

Japan Quince

Purple Fringe -

$$
\begin{aligned}
& 2 \text { " } 3 \text { " } 75 \text { " } \\
& \text { - } 2 \text { "3 } 375 \text { " } \\
& \text { - } \quad 2 \text { "6 } 3 \text { “6 } 75 \\
& \text { - } 2 \text { " } 3 \text { “6 } 75 \\
& 2 \text { " } 3 \text { " } 75 \text { ، } \\
& \text { - } 2 \text { "3 } 375 \text { “ } \\
& 2 \cdot 3 \text { “ } 75
\end{aligned}
$$

\section{Climbing Vines}

Halls Japan Honeysuckle Trumpet Vine -

Boston Ivy

Virg. Creeper

Climbing Roses

Alaska Daisies

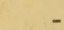

$50 \mathrm{c}$ each
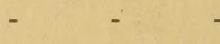